\title{
High-Reliability Organizing (HRO), Decision Making, the OODA Loop, and COVID-19
}

Daved van Stralen, MD, FAAP, Thomas A. Mercer, RAdm, USN

\section{Abstract:}

Organizations do not make decisions; people do. The entropy of any system moves toward disorder of its elements and the corruption of information as it is transmitted. This flux creates uncertainty. As we gain additional information, we find that the property of monotonicity from classical logic and scientific reasoning does not allow us to change our conclusions. We must engage with these situations without a developed plan or even without a plan. Reciprocal decision-making describes how we act, observe the response to our action, and how that response guides our next action. Decision-making with reciprocal feedback keeps the operator close to events, generates information, identifies relevance, and gives meaning to information. This is an active method for generating information, creating structure, and identifying the boundaries of performance. John Boyd's OODA loop is a cycle for rapid, real-time interactive hypothesis development and testing. This is not decision-making independent of others or the organization. The Orient function of Boyd's OODA loop integrates the individual, situation, and organization through culture.

\section{"Organizations do not make decisions;" people do. People do not make errors; the decision does not fit the situation. Failure is not an option; failure is the option that drives engagement."}

\section{Introduction}

Organizations do not make decisions; people do. People do not make errors; the decision does not fit the situation. Failure is not an option; failure is the option that drives engagement.

Operational interpretations of these terms have become washed out for management, planning, and conceptual tractability. Decision, error, and failure have shifted from the concreteness and the continuation necessary for actual-world operations to the abstractions and concepts favored by academicians and spectators. "The advantage of confining attention to a definite group of abstractions is that you confine your thoughts to clear-cut definite things, with clear-cut definite relations," Alfred North Whitehead (1). Once defined, the definition becomes the object.

The privileged role that abstractions, concepts, and cognition have in the organization tacitly corrupts processing information. Operators continuously develop information from threats, and information fluctuates within the changing environment. "Clear-cut definite things, with clear-cut definite relations" do not help make decisions during uncertainty, ambiguity, and threat. Problems arise when our knowledge does not fit our definitions or the situation. We can engage while learning by doing or retreat, identify best practices, and find appropriate rules.

\section{"Science does not press analysis too far beyond the surface of reality into the detailed specifics of life, remaining instead in the world of probabilities, common denominators, concepts, models, and abstractions $(2,3)$."}

Science does not press analysis too far beyond the surface of reality into the detailed specifics of life, remaining instead in the world of probabilities, common denominators, concepts, models, and abstractions $(2,3)$. "Thought deals thus solely with surfaces. It can name the thickness of reality, but it cannot fathom it, and its insufficiency here is essential and permanent, not temporary," William James (4). Abstract generalities are not expert systems (35). Real-life problems are specific and concrete, to be dealt with on their own terms with commonsense problem solving, action, and interaction, experiencing the problem directly in the realm of expertise $(3,4,6,7)$. "Thinking begins first with suggestive but defective plans and images, that are slowly (if ever) refined and replaced by better ones," Marvin Minsky (8).

HROs developed from operations that rapidly "refined and replaced better ones." These are rapidly changing and evolving regions that exist or develop between the rules (protocols, algorithms), when rules (policies) do not apply, or when rules conflict or compete (9). This is where HRO operates. Describing operations for this region means that HRO does not function in opposition to any program. The HRO approach neatly, quietly fits within the organization.

We must not forget that the language of $\mathrm{HRO}$, reliability, and safety is built from interactive decisions and engaged sensemaking. The function of decision-making and sensemaking is to maneuver through changing events while bringing those events to resolution. Error is a product of decision-making under uncertainty (10). Failure is to quit too soon.

Decision-making with reciprocal feedback keeps the operator close to events, generates information, identifies relevance, and gives meaning. The experience of reciprocal feedback forms the sense of agency that staff need to engage uncertainty. Communicating by reciprocal feedback, that is, using statements of "action-

NEONATOLOGY TODAY is interested in publishing manuscripts from Neonatologists, Fellows, NNPs and those involved in caring for neonates on case studies, research results, hospital news, meeting announcements, and other pertinent topics.

Please submit your manuscript to: LomaLindaPublishingCompany@gmail.com 
response," drives a more lucid and objective flow of information in a confusing situation. This form of decision and communication relies on authority migration that allows individuals to make decisions then act on those decisions.

At this point, leaders, executives, administrators, and managers will turn to abstractions, the clarity of defined concepts, and the certitude of cognitive processes. Possibly it is a leap of faith, but reciprocal decision-making to reduce complexity is the hidden foundation of responsiveness and generates communication that forms safety, reliability, and resilience. Without it, HRO becomes another abstract cognitive program that makes sense to central leaders, is measurable and straightforward to teach but comes with diminished functional value.

Decision-making for high reliability is an active method for generating information, creating structure, and identifying the boundaries of performance. The literature on decision-making and error distracts us from the nature of local, individual decision-making in uncertainty, even for mundane, routine activities. The literature misses that, even for routines, we must select a rule or algorithm early on from imperfect and ambiguous information. The fact that many of these situations self-resolve or respond to almost any action can mislead us about the effectiveness of our program. From the most to the least experienced, we must at times choose between conflicting or competing rules or engage in situations that lie between the rules. People will choose appropriate but different rules, or they will make their best decisions between established rules.

\section{"The operator in the flux of a trajectory learns the salience of subtle and nuanced signals, identifies information's relevance and gives meaning to changing information. Experience teaches us to change the level of salience, work with partial or contingent relevance, and shift the meaning of information as events evolve. To the spectator at a fixed point outside of events, these appear as weak signals, irrelevant information, fat-tailed distributions, or random, independent outliers."}

The operator in the flux of a trajectory learns the salience of subtle and nuanced signals, identifies information's relevance and gives meaning to changing information. Experience teaches us to change the level of salience, work with partial or contingent relevance, and shift the meaning of information as events evolve. To the spectator at a fixed point outside of events, these appear as weak signals, irrelevant information, fat-tailed distributions, or random, independent outliers. (In the fat-tailed distribution, the probability of an event in a sigma farther from the mean is greater than the same sigma in the normal distribution. That is, the risk of an event becomes increasingly significant.) To the operator buffeted by events, these same signals represent possible covert, compensated early failures.

Our interactions with failure make visible the early heralds of that failure. Visibility facilitates earlier engagement of subsequent incidents. To respond earlier, however, is to respond to ambiguous signals. While much is made of "weak" signals, these are more likely early, subtle, nuanced, and even punctuated signals in an emergency. The salience, meaning, and relevance of these signals are lost to outsiders.

This article will describe straightforward methods for decision making in the VUCA-2T environment (Volatility, Uncertainty, Complexity, Ambiguity-Threat, and Time Compression, see Table 1) (11), modified from the U.S. Army concept VUCA $(12,13)$ and the anthropological concept of liminality (14). Such situations do not often readily translate into rules, protocols, algorithms, or purely cognitive processes $(10,15,16)$. Inquiry is active and operational, generating information, supporting information flow, and supporting authority migration $(9,16,17)$.

\begin{tabular}{|l|l|}
\hline Volatility & A rapid, abrupt change in events \\
\hline Uncertainty & $\begin{array}{l}\text { Lack of precise knowledge, need for more } \\
\text { information, unavailability of the necessary } \\
\text { information }\end{array}$ \\
\hline Complexity & $\begin{array}{l}\text { A large number of interconnected, chang- } \\
\text { ing parts }\end{array}$ \\
\hline Ambiguity & $\begin{array}{l}\text { Multiple interpretations, causes, or out- } \\
\text { comes }\end{array}$ \\
\hline Threat & Impaired cognition and decision-making \\
\hline Time Compression & $\begin{array}{l}\text { Limitation acquiring information, deciding } \\
\text { or acting before consequential changes }\end{array}$ \\
\hline
\end{tabular}

Table 1. VUCA-2T (11)

Decisions are how we engage real-life problems in the VUCA-2T environment or during ordinary and mundane operations. What is expected by the veteran is novel to the beginner, yet the decision processes for refining and replacing plans are the same. Decisions must be timely; we must form mental concepts for the situation and change these concepts as the situation changes. "The concepts can then be used as decision models for improving our capacity for independent action... How do we generate or create the mental concepts to support this decision-making activity?" John Boyd (18). Reciprocal decision-making to reduce complexity is a method facile to the beginner and elegant for the veteran.

\section{The Embedded Problem}

At first, observation is all we have. Our engagement of a situation starts with our first look when we let our looking teach us. With experience, we gain the "art of describing in darkness and through the fog" (5). The inclusion of uncertainty widens our operational environment making available a fuller spectrum of analysis and the pursuit of weak but salient signals (19).

\section{"The inclusion of uncertainty widens our operational environment making available a fuller spectrum of analysis and the pursuit of weak but salient signals (19).",}

Uncertainty as an advantage appears counterintuitive. When we encounter a new situation, it seems advantageous to select a similar frame $(8)$ or draw upon a rule or maxim $(20,21)$. Our choices 
bring expectations, adding difficulties if the frame does not accurately match the situation. An organization or industry may institutionalize competency over proficiency because generalizable principles can be taught to a high-turnover, novice workforce (21). Consequently, the most likely person to encounter an emerging problem or new situation may be a relative novice who will think from rules.

\section{"The entropy of any system moves toward disorder of its elements and the corruption of information as it is transmitted (22). This flux creates uncertainty."}

The entropy of any system moves toward disorder of its elements and the corruption of information as it is transmitted (22). This flux creates uncertainty. Our observations will shape the concepts we formulate and shape our observations and inquiries (18). Accepting uncertainty and incompleteness of our concepts reduces surprises (8). Boyd posited that "according to Gödel, we can not-in general-determine the consistency, hence the character or nature, of an abstract system within itself." To rely solely on established concepts is to operate within the system. Boyd described the need to use analysis to differentiate elements while using synthesis with outside information to integrate a solution outside the system (18).

The organization with a 'rule-error' focus may force the expert to accept concepts and follow the rules. The expert will then perform poorly, at a lower level than the novice $(20,21,23-25)$. We also risk surprises when we must use the "best-fit frame (8). Dynamic conjunctions of facts have different relations than we can predict or expect. Events may abruptly change trajectory. Our actions cause punctuated responses. When we have fewer expectations, we create fewer surprises, lowering the barrier to earlier engagement.

Organizations seeking to increase reliability and decrease error look to cognitive processes and classical problem-solving. These methods utilize deductive analysis, scientific logic, and critical thinking. Unintentionally, this supports certainty, disregards ambiguity, and supports deterministic, linear problem solving, more like puzzle-solving where knowable information fits together to produce the right answer $(19,26)$.

\section{The limits of logic and science}

"Thought deals thus solely with surfaces. It can name the thickness of reality, but it cannot fathom it, and its insufficiency here is essential and permanent, not temporary," William James (4). Classical logic and scientific reasoning fail the operator faced with an unfamiliar situation (27).

Classical logic and scientific reasoning do not allow additional information to invalidate conclusions, the property of monotonicity $(8,28)$. Enforcement of classical logic and monotonicity explains why we may consider a person who changes their stance as "flipflopping" or "waffling" rather than someone who revises beliefs from new or updated information. The principle of consistency in classical logic does not allow contradictions $(29,30)$. "Logical reasoning is not flexible enough to serve as a basis for thinking... The consistency that logic absolutely demands is not otherwise usually available - and probably not even desirable!-because consistent systems are likely to be too 'weak,"' Marvin Minsky (8).
Scientific theories preserve order by putting a name to some observed object, subsuming the observation within a known object (2), and organizing findings into logical, compartmentalized categories of knowledge $(6,31)$. "When we conceptualize, we cut out and fix, and exclude everything but what we have fixed. A concept means a that-and-no-other," William James (4).

In the typical situation, the operator must find an analogy between what is explored and known (2), does not know what facts are relevant, uses knowledge not previously thought to be relevant, and revises knowledge even as the operator uses that knowledge (31). A gap begins to form "between the apparent reality and one methodically ascertained; between phenomena in their concreteness and particularity and facts stripped of all that is tangible and individual so that they may be fitted into some all-embracing regularity," Siegfried Frederick Nadel (2).

James described "thought" as solely dealing with surfaces. Nadel (2) describes how science does "not press their analysis very far beyond the 'apparent' reality and into an order of things where waves of probability undulating into nothingness offer the ultimate common denominators... The human sciences are concerned with the phenomenal surface of reality; if they discarded it immediately, they would destroy their subject matter."

\section{Action without a plan}

It was common to engage in a situation without a developed plan. Less typical was to engage without any plan. One author (DvS) discussed this with members of a special group in SOCOM (Special Operations Command) that to engage without a plan was to accept that "failure is an option." Plans can lead to adherence and disregard of anomalies and failures in operations.

\section{"It was common to engage in a situation without a developed plan. Less typical was to engage without any plan. One author (DvS) discussed this with members of a special group in SOCOM (Special Operations Command) that to engage without a plan was to accept that "faillure is an option." Plans can lead to adherence and disregard of anomalies and failures in operations."}

The "phenomenal surface of reality" is our first observation, all that we have. "The only way in which to apprehend reality's thickness is either to experience it directly by being a part of reality one's self or to evoke it in imagination by sympathetically divining someone else's inner life," William James (4). Our engagement of a situation starts with our first look, it is our "looking" that teaches us. Only action by interaction can change or remake objects.

The "primary purpose of problem-solving is to understand the problem space better," Marvin Minsky (8). The belief that knowable information enhances decision-making contributes to collecting more information and increases confidence but with little change in accuracy (26). Rather than a puzzle solved by identifiable, knowable information, we can approach the problem as a mystery where generated information generates new decisions 
(19). Loop decision-making is practical, providing answers to what works rather than why or how the answer came about. Commonsense decision-making deals with every concrete situation on its own terms (6). "Thinking begins first with suggestive but defective plans and images, that are slowly (if ever) refined and replaced by better ones," Marvin Minsky (8).

"[I]ntelligence is incarnate in overt action, using things as a means to affect other things. Thought, reason, intelligence, whatever word we choose to use, is existentially an adjective (or better an adverb), not a noun. It is the disposition of activity, a quality of that conduct which foresees consequences of existing events, and which uses what is foreseen as a plan and method of administering affairs," John Dewey (32). When we foresee the consequences of our situation, consequences of acting and not acting, then those consequences form our plan, and they direct our decisions.

\section{"When we foresee the consequences of our situation, consequences of acting and not acting, then those consequences form our plan, and they direct our decisions."}

\section{Complexity}

Complexity at a distance seems to have sufficient organization for spectators to discuss forms, structures, and causes. Complexity up close gives the operator few options for engagement. Ensuring safety through distance from the threat seems more reliable than safety through engaged operations. Well-thought-out decisions have greater effectiveness than hasty actions. Maybe this is because "a story always sounds clear enough at a distance, but the nearer you get to the scene of events, the vaguer it becomes" George Orwell, Shooting an Elephant (33). These are the paradoxes and contradictions of the VUCA-2T environment.

Complexity and chaos appear identical to the operator. Uncertainty generates information (22). Ambiguous information drives investigation (34). Actions create equipoise (35). The only certainty is learning by doing. With a far more limited spatial and time horizon than the spectator, the operator can only experience relations and movements. The complexity comes from a large number of elements in linear relations. Chaos develops from a few elements with nonlinear relations but at a high rate of change (36).

The operator enters the VUCA-2T situation by reducing linear relations and slowing the rate of change. The reduction of complexity makes the problem's structure visible. The slower rate of change decreases the constant presentation of randomness. Friction and failure to respond create negative space around the threat, generating a better understanding of demands (5). This approach favors engagement over assessment. Approaching an emergency, a Los Angeles City firefighter said, "I don't know what's happening, but I know what to do."

From the normative stance outside the flow of events, uncertainty, complexity, chaos, and randomness are independent threats. They are the operating environment from the pragmatic stance within the trajectory and experiencing the flux of events. Common sense differentiates these approaches (7): "The motions of a round-about [British, merry-go-round] are only puzzling until we lift the trapdoor and discover a well-known engine driving the thing. Common sense always urges us to look for such trapdoors," Nadel (2). The reciprocal decision-making of common sense is how we lift trapdoors.

The surface of reality, what we observe and can more readily measure, is the trapdoor concealing the mechanism of the problem. It particularly confounds the novice and new residents in the ICU. Numerous active critical care problems can make the initial presentation of a patient difficult. The author (DvS) would list all information on a whiteboard, no matter how insignificant. The first items mentioned were always fluids, electrolytes, and nutrition ("FEN"), active tasks for all patients in the hospital, therefore, most available to the resident's mind. We routinely developed four columns of seven to ten items. We crossed out information duplicated because of separate means to measure the same thing. We grouped the remaining information by physiological system or treatment modality, producing four to five groupings. We identified what could kill the patient, what would prevent the transfer to the hospital ward, and routine problems from this list. We generally found only two problems were deadly. Changing from a complex situation to a few deadly problems allowed the residents to focus on the one or two life-threatening problems and feel assured the routine problems would continue to be addressed.

This approach came about from an early resuscitation in the NICU during the author's internship. The author, as a former paramedic, was deciding whether to intubate the infant. The senior resident rushed in and immediately called for a flashlight. Intrigued, as neonatology was new to the author, he watched as the senior resident transilluminated the scrotum and loudly diagnosed hydrocoele. When the Neonatologist arrived, the author was working on the connection between the scrotum, hydrocoele, lack of chest wall movement, and how to fix it. We proceeded with intubation. This central problem, repeated throughout the author's experience in healthcare, was how people would first address the problem they knew.

Working with concepts is to focus on the surface of the problem rather than its thickness. The individual is then separated from the problem. Decisions will emanate from decontextualized thought and the inability to perceive subtle or nuanced early heralds. In the $\mathrm{HRO}$, thought, described by Dewey (32) as an adverb, modifies decision making, enabling us, as described by William James, to directly experience and apprehend reality's thickness (4).

We experience complexity and chaos during VUCA-2T. Concepts inform but do not define the thought that modifies decision-making. To solve the problem, we must better understand the problem space (8) and feel the texture of events. We become part of the problem and the texture of the environment. That is how you solve the problem - we make decisions to change the texture. We change texture from within.

\section{Approaching a situation}

At our first look, without expectations or surprises, all problems appear to be complex and chaotic. Duncan Dieterly (37) described the three elements of a problem: the situation, intervention, and objective. Knowing all three makes the problem trivial, efficiently dealt with using rules and protocols. Because the situation defines the problem (Dieterly's "defined problem"), we can select the appropriate decision tree, algorithm, or clinical pathway when we know the initial situation. This computation now describes much of healthcare's efforts for safety, error management, and reliability.

The more intractable problem is the uncertain or unknown situation, classified by Dieterly as the intuitive or undefined problem. It is the basis of this paper.

We approach these situations by developing a concrete objective. Dieterly's work, elaborated by George Orr (38), describes con- 
ditions with multiple situations, interventions, and objectives. At first, this may appear confusing, but by correlating these conditions with your experience, you readily elaborate decision-making for complex, chaos-driven situations.

Information collection. We urge caution when collecting information to justify a decision. The team may follow their focus, disregarding or discounting any disconfirming information. Lack of support from the organization or leadership creates a sense of never having sufficient information to act. A more readily addressed problem is the difficulty in documenting indications to support a decision.

Multiple situations. The novice may see one situation: everyone in turmoil. Through observation, modeling, and experience, events begin to settle, and multiple situations are identified. The Neonatologist will identify more subtle situations, such as failure to respond to treatment, an unexpected finding, or some anomaly. Distinguishing such situations facilitates assigning appropriate team members, providing greater clarity and purpose for authority migration.

Multiple interventions. We commonly teach novices a single intervention. Rotating with different Attendings, medical students and residents learn different treatments. Rather than a point of value judgment toward those who teach, the student can benefit from this. The RCPs at a subacute facility refused to participate in rounds with the author (DvS). The respiratory manager inquired and received the complaint that they answered the author's question, then the next week, that answer was incorrect. The following week was the same experience; now, both answers were incorrect. The manager explained that the author did not want them to think there is only one answer. He wanted them to have 3-4 ways to treat any problem because their patient will be dying one day, and their regular treatment would not work. Knowing multiple ways to treat became a matter of pride when the RCPs would teach students.

"He wanted them to have 3-4 ways to treat any problem because their patient will be dying one day, and their regular treatment would not work. Knowing multiple ways to treat became a matter of pride when the RCPs would teach students."

Multiple objectives. Physiologically we address cardiac output, respiratory function, neurologic function, and nutrition. However, during an emergency, we also monitor stress-fear-threat effects in our staff to ensure optimal performance. If parents are present, we make visible our physical efforts to save their baby. We have the objectives and interventions to increase success, but we add the necessary objectives and interventions to reduce failure. Less experienced participants often miss this as they focus on approaching success. Finally, and quite delicately, we have the objective close to our heart for the parents. Should their baby die, the parents feel that everything was done for their baby and that the staff know they love their baby. All parents want us to know how much they love their baby.

Decompose objectives. George Pólya described a problem-solving heuristic of varying the problem by decomposing and recombing its elements or use of analogy (39), methods also found in practical common sense problem solving (7). When we cannot always reach our objective, we can rapidly decompose the objective to 'sub-objectives.' For example, Airway and Breathing are decomposed objectives to achieve respiratory function.

Not reaching an objective can initiate stress-fear-threat responses and further degrade decision performance. If staff cannot identify a solution, they may become frustrated. With repeated occurrences, staff become susceptible to learned helplessness or loss of agency.

The Neonatologist can decompose objectives at multiple points, from teaching and planning through active resuscitation when situational problems become evident. When decomposed during training, the student can amalgamate steps in a way that makes sense to them. A dance instructor learned this from the author (DvS). Rather than the standard to guide how to amalgamate dance steps, the instructor told the student to decide how to amalgamate steps. Specialized training in rope work for special groups in SOCOM (Special Operations Command) now uses this approach, significantly increasing learning.

Decomposed objectives facilitate authority migration. The Neonatologist can decompose larger objectives to a set that various operators can achieve within their capabilities and scope of practice. This capability also forms the basis of the Incident Command System used by the fire service $(40,41)$ and is now a part of hospital disaster response.

\section{"Decomposed objectives facilitate authority migration. The Neonatologist can decompose larger objectives to a set that various operators can achieve within their capabilities and scope of practice. This capability also forms the basis of the Incident Command System used by the fire service $(40,41)$ and is now a part of hospital disaster response."}

Uncertainty. Here, we discuss uncertainty in the information itself. Uncertainty is embodied in the presentation. Heisenberg's Uncertainty Principle arises from the "complementarity principle" that describes how the measurement of one property requires measurements from the other complementary property. For example, a particle's position requires the value of the momentum, and the particle's momentum requires the value of the position. This property is inherent to wave-like systems such as those with linear-time variance. There is a spread in time and frequency; you cannot have substantial limitations in both or know both simultaneously. We can encounter these relationships during an active resuscitation, such as collecting information (frequency) that takes time. In an active event during a VUCA-2T incident, the situation has changed in the time to collect information for precision.

\section{Reciprocal decision making}

Reciprocal decision-making describes how we act, observe the response to our action, and how that response guides our next action. We learn what works through action. Decisions linked to action are probes to learn structure, redirect trajectory, create 
structure, and engage the threat. This decision is not trial-by-error or random action but focused on the objective and observed situation. It is also not simple feedback, a component of decision trees or algorithms.

You identify reality by the way it responds to you. While abstractions do not respond to your actions, many people do respond to abstractions. This context differentiates reflective thought from thinking by acting and motor cognition. Acting gives constant feedback for updating and revision of beliefs.

\section{"It is dangerous during an emergency to think that people from different socio- economic statuses make their decisions differently. More likely, their decisions are influenced by stress and fear and are more alike than is recognized (42). Inexperienced or untrained people are equally likely to make decisions for the good of others."}

It is dangerous during an emergency to think that people from different socio-economic statuses make their decisions differently. More likely, their decisions are influenced by stress and fear and are more alike than is recognized (42). Inexperienced or untrained people are equally likely to make decisions for the good of others.

Accuracy versus Precision. "Ready, fire, aim!" Army artillery cannot use precision when their target is not ideally placed. Instead, they use 'bracketing' to increase their accuracy by firing, taking a measure, re-aiming, and firing again. Their rounds hit increasingly closer to the target. We achieve accuracy through feedback with the environment, changing our actions as we observe how close we are to our objective. Precision initially may seem more desirable, but it is an internal measure independent of the environment yet will be affected by it. Repeated actions will not reliably reach the target. In precision, we hit the same point repeatedly, even if we miss our target. Accuracy allows us to change with the situation, coming closer to the target each time.

Feedback. We have immediate, short, long, delayed, and indirect feedback loops. We select actions from past experiences to gain exploitation by making new choices for exploration and through observation for growth or safety. Environmental responses must be observable and salient if they are to reinforce behavior and lead to learning. This response is HRO trial-and-error learning. In this way, the environment acts upon the individual and organization described by Bob Bea, Professor Emeritus, Civil Engineering, University of California, Berkeley (43), as interactive, real-time risk assessment and management.

Short feedback loops are more specific to our actions and more readily accepted, rightly or wrongly, as causative. For example, instrumental anger or intimidation to gain control of staff has a short feedback loop used for that effect in movies. The long and indirect feedback is disastrous and may be hidden, such as in high staff attrition.

The time compression inherent to the flux of rapid, dynamic events confounds the real-time use of prolonged or indirect feedback loops. To obtain short feedback loops, we must closely approach the environment, even entwining with the environment. Entering the situation shortens feedback loops, thus improving accuracy.

Negative and positive feedback. Feedback maintains homeostasis for stable operations within a VUCA-2T environment, supports safety, generates self-organization WHILE simultaneously bringing resolution to the event. Negative feedback corrects deviations from our desired state, and positive feedback supports our strength and resilience. To an outside observer, this method of homeostasis may appear to be one of constant or repeated error rather than continuous assurance of effectiveness and safety.

A control system maintains homeostasis by monitoring distance from a set point. Negative feedback is a deviation from the desired set point that can be offset by corrective action. Negative feedback marks our boundaries for safe operations.

\section{"Negative feedback correction may be classified as an error, but it is a mark of safety, resilience, and adaptability. As we operate in this unstable environment, we will also test the boundaries between our performance capabilities and the limits for safety and harm."}

Negative feedback correction may be classified as an error, but it is a mark of safety, resilience, and adaptability. As we operate in this unstable environment, we will also test the boundaries between our performance capabilities and the limits for safety and harm. These boundary checks can sometimes only be performed in real-world situations in real-time. Failures are helpful in these situations because they mark our performance boundaries. The outsider, not appreciating the exigencies of the situation, may have a different, negative interpretation of this level of operation.

Self-organization. Amplification of positive feedback causes growth and pattern formation, which is calibrated by the stabilizing effects of negative feedback for safe boundaries.

Improvisation. Negative feedback increases the safety of improvisation and, through recalibration, reduces the effect of noise (Weick, personal communication).

Outliers. Outliers in statistics are a tiny population of random, independent values. Outliers in probability are very rare outcomes. The events we work with emerge from routine operations, even if our routine is an extreme cardio-respiratory failure. Events may grow from the routine, creating a power distribution where large events are rare, or from stochastic probability distributions; that is, the event follows a probability distribution, but the distribution changes.

In a series of short feedback loops, people develop sensemaking and create more easily transmitted information. Communicating information as action-response carries information. Describing what the person believes is happening or stating what they believe should be done carries less meaningful information.

\section{The OODA Loop}

Loop decision-making, like common sense problem solving, is practical and drives interaction. It provides answers to what works 
rather than why or how the answer came about.

"Our [dangerous] work has termed this interactive-realtime assessment and management of risks. This approach was completely overlooked until the early 1990 s. We were taught that there was only proactive (before operations) and reactive (after) - and that was it. And we thought we could capture all of the risks with the proactive approaches - and then provide adequate defenses if 'justified' - but we were missing some really major risks that were fundamentally unpredictable and unknowable."

Bob Bea, 8/30/2005, personal communication

In 1968, John Boyd presented his concept of the OODA loop (Figure 1) during the Vietnam War for fighter pilots to outmaneuver their opponents (44). After studying air-to-air combat in the Korean conflict, he developed this approach to better understand why American pilots in Korea flying the F-86, a plane inferior to the MiG-15, had better kill ratios than American pilots in the Vietnam War planes superior to the MiG. He found the difference to be that the American pilots in Korea had better ability to see out of the cockpit and switch from one activity much more quickly to another. We use the OODA loop in healthcare to outmaneuver a rapidly changing disease state (45).
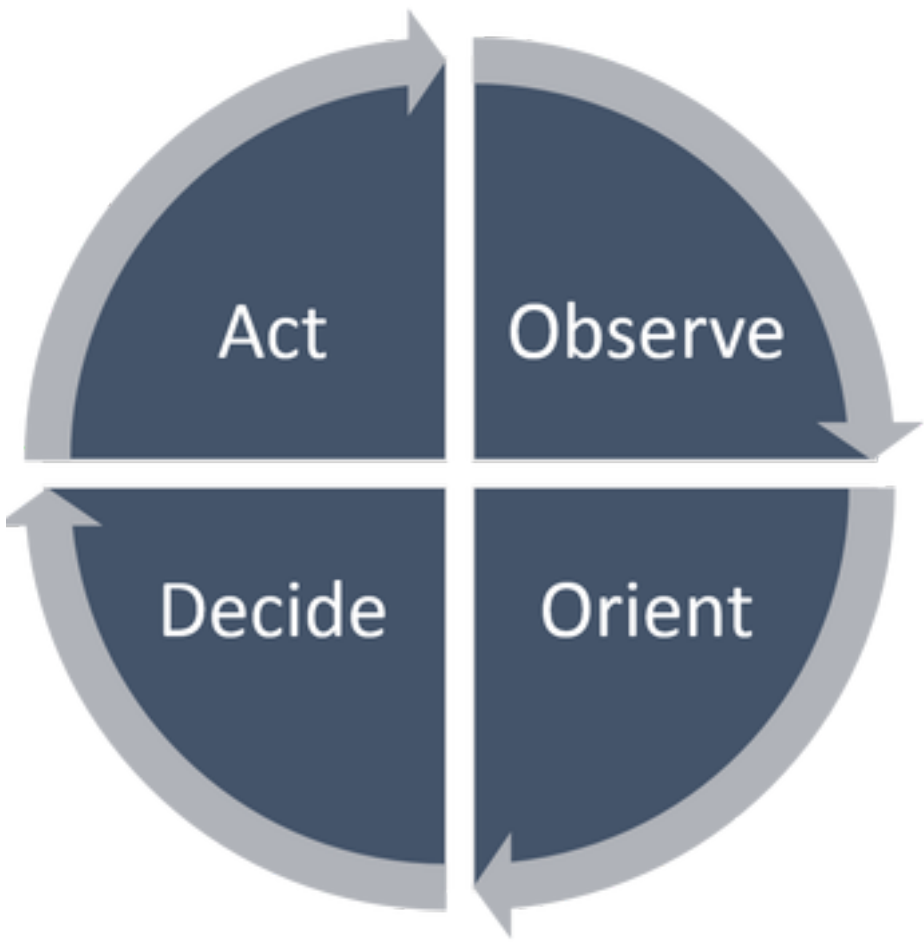

Figure 1. Boyd's OODA loop

Attribution 2.0 Generic (CC BY 2.0)

The OODA loop is a cycle for rapid, real-time interactive hypothesis development and testing. To create the loop, the person $\underline{\mathrm{O} b}$ serves, Orients, Decides, and Acts then loops back to observe the effect of the action. The action creates the loop for a continuous cycle and gives the OODA loop its power and agility.

There need not be a consistent starting element. Weick observed that "one can start this sequence anywhere and move either in a clockwise or counterclockwise direction" (personal communication). Weick has described Boyd's OODA loop (in either sense- making or enactment) by starting at $A$, the "act" phase (personal communication). Acting is the first step in engagement and is the active part of Weick's sensemaking; acting is also the initiator of Weick's enactment. Acting can create both visible and correctable failure through looping. The latter is critical in HRO to oppose failure from not acting, which is invisible, not detectable, and not correctable.

We think by acting (35); that is, we make sense of the situation by acting and gaining positive and negative information, and we enact change in the environment by acting (46).

Observe. An attention function for discrepancies, disruptions, and outliers does not require a complete analysis of the situation. The observe function also notices the responses to our just-completed action and whether we received the results we expected. The system can improve, in which case we may continue; the system may deteriorate, in which case our actions generate new information about its structure, or the system may not change. This last option is dangerous because we might have the wrong dosage, drug, or diagnosis.

\section{"The observe function also notices the responses to our just-completed action and whether we received the results we expected. The system can improve, in which case we may continue; the system may deteriorate, in which case our actions generate new information about its structure, or the system may not change."}

Orient. "Shapes the way we interact with the environment...The way we observe, the way we decide, the way we act." "Seen as a result, represents images, views, or impressions of the world shaped by genetic heritage, cultural tradition, previous experiences, and unfolding circumstances," John Boyd (44). Also, updated information; and analysis and synthesis from feedback, guidance, and control. In this function, we synthesize new information and build support for our decisions. (Some consider this an orientation to the circumstances, but the orientation function is much richer.) This emphasis on orientation makes conflict into a learning contest to better maintain awareness of the world; one can create opportunities to act; orientation of group members (47)

Decide. Generate a hypothesis. The circumstances operate in the affective domain of emotion, values, attitudes, and personal evaluation. Create new repertoires (47).

Act. Initiates the loop action, which acts as a control mechanism operating on the environment while changing people's perceptions of the environment. Using the loop action is a method to explore the situation while exploiting any path of least resistance. When we encounter friction as part of the feedback, we can observe whether we should pursue another path with less friction or identify the friction point as something significant that should remain in focus (38). The use of positive feedback through the loop action directs focus and actions toward paths of success. In contrast, negative feedback marks practical and safety boundaries that can act as a safety function. 
Loop. We calibrate our mental model with actuality and responsiveness of the environment. Encountering resistance or friction, we rapidly resolve whether to change our approach or seek resources. This resolution is not through speed or shortcuts but by increasing our tempo. Boyd described the methods and benefits of a faster tempo: the ability to transition more rapidly than events change, develop more repertoires of action, free and open communication, interactive support, increased information sources to select from, and generation of new ideas that can be rapidly tested (48).

\section{Table 2. OODA Loop functions}

\begin{tabular}{|l|l|}
\hline Function & Description \\
\hline Observe & $\begin{array}{l}\text { Observe the situation } \\
\text { Match prediction to response }\end{array}$ \\
\hline Orient & $\begin{array}{l}\text { Process and synthesis of observations } \\
\text { Actual world function } \\
\text { Culture, experience, physiology }\end{array}$ \\
\hline Decide & Develop hypothesis; decide the action \\
\hline Act & $\begin{array}{l}\text { Test hypothesis } \\
\text { interface between operator and environment }\end{array}$ \\
\hline Loop & Effect of action \\
\hline
\end{tabular}

Connecticut Paramedics

Two paramedics arrived on the scene to find a woman in her late sixties lying face down in the street. They saw she was nonresponsive and pale. Her skin felt damp, and her oxygen saturation was in the low eighties, with a slow, weak heartbeat in the forties. On the EKG, they identified a regular electrical pattern except for premature ventricular contractions. The first thought was that she had had a cardiac event, so they considered cardiac protocols. The two paramedics were unsure which protocol to use, but each paramedic had 15 years of experience and could see that she was dying. They began to feel helpless to stop her death.

\section{"The two paramedics were unsure which protocol to use, but each paramedic had 15 years of experience and could see that she was dying. They began to feel helpless to stop her death."}

While accomplishing this evaluation, they made a rapid visual respiratory exam and observed very shallow breathing; they identified lower airway obstruction from the prolonged expiratory phase. This indicated treatment with a bronchodilator drug, something they would not have considered without the rapid five-point visual respiratory exam (49). However, they were not sure if they should treat what could be a second disease, if the bronchodilator would work, or if the bronchodilator might stimulate the heart and cause the extra beats to fibrillate, creating cardiac arrest.

They decided to administer the drug and observe the response, which is something they would not have considered without the use of Boyd's OODA loop decision-making. After the bronchodilator treatment, the woman's chest expansion increased, the expi- ratory phase shortened, her oxygen saturation increased above 90 percent without supplemental oxygen, and she became alert. Most critically, her heart rate increased, and the premature ventricular contractions resolved. This woman's life was saved by treatment the paramedics had learned four months earlier.

\section{Steep Authority Gradients}

Whether intentional or assumed, healthcare has steep authority gradients. This gradient may not pose a significant problem for routine situations, but it leads to poor dynamics in time-compressed states or information that disconfirms the physician's beliefs. Bedside caregivers will commonly resolve this discrepancy by "adjusting" information to be congruent with the physician's views, withholding information that disconfirms the physician's beliefs, or withstanding personal attacks on their abilities.

The OODA loop can be used for communication as action and response. Presenting only observation (the observe function) relies on credibility. Presenting only the decision (the decide function) is also problematic when we view decision-making as a process of weighing subjective observations, objective data, indications, and contraindications. Instead, by modifying the approach used by fire departments, the caregiver will describe the action taken (act function) and the response observed (looping to a new observe function). This loop brings focus to the disease process and interventions rather than personal biases and credibility.

\section{"Instead, by modifying the approach used by fire departments, the caregiver will describe the action taken (act function) and the response observed (looping to a new observe function). This loop brings focus to the disease process and interventions rather than personal biases and credibility."}

\section{The decision frame}

Faced with a situation having an uncertain, possibly harmful, outcome, we may hesitate to act. A decision frame can bring focus to the consequences of acting or not acting. To create the frame, ask what happens if you act too soon or too late. Then ask what consequences are likely to happen if your intervention is too little or too much.

The licensed vocational nurse (LVN) wanted fluids through the gastrostomy tube to treat thick pulmonary secretions. We had two discussions, one for treating the secretions and one regarding the fluid. "What would happen if we didn't treat the secretions, or if we treated them and didn't need to? And what would happen if we gave too much or not enough fluid?"

She answered that treating the secretions too early would thin secretions and increase the urine output. Waiting to treat the secretions could lead to the formation of thick mucus plugs, an obstructed airway, and cardiac arrest. For the amount, giving too much would increase the urine output, while giving insufficient amounts would delay response and increase the threat of thick secretions, which could lead to cardiac arrest. She realized that, within her decision frame, her decisions would lead to more urine or death. The LVN became vigilant for secretions and better un- 
derstood what we were treating, how we treated it, and why we make the decisions we do.

\section{"We can reverse the administration of too much fluid with a diuretic. This problem arises when we titrate fluids for dehydration or blood volume and, though we can calculate ideal weight and expected blood volume, we still do not know precisely how much fluid would benefit our patient."}

Is the decision reversible? Often, we find the novice decisionmaker worrying over an action that can readily be reversed. For example, we can reverse the effect of a diuretic with the administration of fluid. We can reverse the administration of too much fluid with a diuretic. This problem arises when we titrate fluids for dehydration or blood volume and, though we can calculate ideal weight and expected blood volume, we still do not know precisely how much fluid would benefit our patient.

Another critical factor in the decision frame is the outcome, whether good, bad or no change. If it is good, then we continue doing it. We do not express it as a poor decision if it is bad but as new and vital information. A harmful or unwanted result identifies the boundary of our envelope for operational purposes and helps us identify structure within what initially appears to be an unstructured situation.

The most dangerous outcome is "no change." In the medical field, we may have the wrong dose, wrong drug, or wrong diagnosis. "No change" increases our uncertainty and supports more vigorous attempts to characterize the structure of our problem.

The most critical decision is the decision "not to act." This "nonacting" can signal to others that no action is necessary or that the problem is not severe. Observation, the key to "not acting," may be passed over. The leader must reiterate that "the decision not to act is a decision."

\section{"In the medical field, we may have the wrong dose, wrong drug, or wrong diagnosis. "No change" increases our uncertainty and supports more vigorous attempts to characterize the structure of our problem."}

\section{Conclusion}

The central issue for the HRO is the embedded problem -- where the entropy of the system moves toward the disorder of its elements and information is corrupted by its transmission. Individuals find themselves in situations where they must act without a plan, working from rapidly identified objectives. Decomposing dif- ficult to reach objectives while simultaneously working on multiple objectives is made possible by reciprocal decision making. The decision changes the environment, which then changes the next decision.

This is not making decisions independent of others or the organization. The Orient function of Boyd's OODA loop integrates the individual, situation, and organization through culture.

Dynamic, complex problems are specific and concrete that we deal with on their terms, not ours. We interact to learn what works while simultaneously ensuring safety. We achieve this, not through plans or rules but reciprocal feedback. We create our expertise through our own experience and from watching others.

We gain that experience through interaction with the problem, the situation, and the environment (50). Perhaps it is the function of decision-making and sensemaking to allow us to maneuver through these changing events.

Decision making with reciprocal feedback has multiple functions:

the operator works close to, even within, events,

uncovering information and generating Shannon Information,

identify the relevance of information, give meaning, and reduce ambiguity,

contributes to the sense of agency needed to engage uncertainty,

communication through "action-response," and

drive the lucid and objective flow of information in a confusing situation.

Decision-making for high reliability is an active method for generating information, creating structure, and identifying the boundaries of performance.

"Decision-making for high reliability is an active method for generating information, creating structure, and identifying the boundaries of performance."

"Operators are maintained in [complex technological] systems because they are flexible, can learn and do adapt to the peculiarities of the system, and thus they are expected to plug the holes in the designer's imagination," Jens Rasmussen (51).

\section{References:}

1. Whitehead AN. Science and the modern world. Cambridge, UK: Cambridge University Press; 1926.

2. Nadel SF. The Foundations of Social Anthropology. London, UK: Routledge; 1951.

3. de Kleer J, Brown JS. A Qualitative Physics Based in Confluences. In: Hobbs JR, Moore RC, editors. Formal theories of the commonsense world. Ablex Series in Artificial Intelligence. 1. Norwood, NJ: Ablex Publishing Corporation; 1985. p. 109-84.

4. James $W$. Essays in radical empiricism [and] $A$ pluralistic universe. Gloucester, MA: P. Smith; 1912. 
5. van Stralen D, Mercer TA. The Art of Neonatology, the Art of High Reliability as a Response to COVID-19. Neonatology Today. 2021;16(2):74-83. doi: 10.51362/neonatology. today/202121627483.

6. Watts DJ. Everything is obvious *Once you know the answer: How common sense fails. London, UK: Atlantic Books; 2011.

7. van Stralen D, Mercer TA. Common Sense High Reliability Organizing (HRO) in the Response to COVID-19. Neonatology Today. 2021;16(7):90-102. doi: 10.51362/neonatology. today/2021716790102.

8. Minsky M. A framework for representing knowledge. In: Winston PH, editor. The Psychology of Computer Vision. New York, NY: 211-277; 1975.

9. van Stralen D, McKay S, Mercer TA. Flight Decks and Isolettes: High-Reliability Organizing (HRO) as Pragmatic Leadership Principles during Pandemic COVID-19. Neonatology Today. 2020;15(7):113-21. doi: 10.51362/neonatology.today/20207157113121.

10. van Stralen D, Gambino W. Error as a Faulty Failure Signal. Neonatology Today. 2020;15(9):114-7. doi: 10.51362/neonatology.today/20209159114117.

11. van Stralen D, Mercer TA. High-Reliability Organizing (HRO) in the COVID-19 Liminal Zone: Characteristics of Workers and Local Leaders. Neonatology Today. 2021;16(4):90-101. doi: 10.51362/neonatology.today/2021416490101.

12. Arnold III AV. Strategic visioning: What it is and how it's done. Carlisle Barracks, PA: United States Army War College, 1991.

13. Magee RR. Strategic leadership primer. Carlisle Barracks, PA: United States Army War College, 1998.

14. Szakolczai A. Liminality and experience: Structuring transitory situations and transformative events. International Political Anthropology. 2009;2(1):141-72.

15. van Stralen D. Pragmatic High-Reliability Organization (HRO) During Pandemic COVID-19. Neonatology Today. 2020;15(4):3-9.

16. Dewey J. The pattern of inquiry Logic: The Theory of Inquiry. New York, NY: Holt, Rinehart and Winston; 1938. p. 101-19.

17. Weick KE. Sensemaking in organizations. Dickens $G$, editor. Thousand Oaks, CA: Sage; 1995.

18. Boyd J. Destruction and creation. Fort Leavenworth, KS: US Army Comand and General Staff College, 1976.

19. Wolfberg A. Full-spectrum analysis: A new way of thinking for a new world. Military Review. 2006;86(4):35-42.

20. Dreyfus SE, Dreyfus HL. The Scope, Limits, and Training Implications of Three Models of Aircraft Pilot Emergency Response Behavior. Berkeley, CA: University of California, Berkeley, Center OR; 1979 February 1979. Report No.: ORC 79-2 Contract No.: ORC 79-2.

21. Benner P. From Novice to Expert: Excellence and Power in Clinical Nursing Practice. Menlo Park, CA: Addison-Wesley; 1984.

22. Shannon CE. A Mathematical Theory of Communication. Bell System Technical Journal. 1948;27(3):379-423. doi: 10.1002/j.1538-7305.1948.tb01338.x.

23. Dreyfus HL, Dreyfus SE. The Psychic Boom: Flying Beyond the Thought Barrier. Berkeley, CA: University of California, Berkeley, Center OR; 1979 March 1979. Report No.: ORC 79-2.

24. van Stralen D, McKay S, Williams GT, Mercer TA. Tactical
Improvisation: After-Action/ Comprehensive Analysis of the Active Shooter Incident Response by the San Bernardino City Fire Department December 2, 2015. San Bernardino, CA: San Bernardino County Fire Protection District; 2018.

25. De Maio J, Parkinson S, Leshowitz B, Crosby J, Thorpe J. Visual Scanning: Comparisons Between Student and Instructor Pilots. Brooks Air Force Base, Texas: Air Force Systems Command, 1976 Contract No.: AFHRL-TR-76-1.

26. Heuer RJ, Jr. Psychology of Intelligence Analysis. Langley, VA: Center for the Study of Intelligence, CIA; 1999.

27. van Stralen $D$, Mercer TA. High-Reliability Organizing (HRO) and Abrupt Change from COVID 19: Failure of Scientific Rationality and Classical Logic. Neonatology Today. 2021;16(6): 97-109. doi: https://doi.org/10.51362/neonatology.today/2021616697109

28. McCarthy J. Some expert systems need common sense. Ann N Y Acad Sci. 1984;426:129-37. Epub 1984/01/01. doi: 10.1111/j.1749-6632.1984.tb16516.x. PubMed PMID: 6391331.

29. Priest G. Paraconsistent logic. In: D.M. G, F. G, editors. Handbook of philosophical logic. 6. Second ed. Dordrech, Germany: Springer; 2002. p. 287-393.

30. Da Costa NC. On the theory of inconsistent formal systems. Notre Dame Journal of Formal Logic. 1974;15(4):497-510.

31. McCarthy J. Artificial intelligence, logic and formalizing common sense. In: R.H. T, editor. Philosophical logic and artificial intelligence. Berlin, Germany: Springer, Dordrecht; 1989. p. 161-90.

32. Dewey J. Experience and nature. Dover, NY: Courier Corporation; 1958.

33. Orwell G. Shooting elephant. In: Packer G, editor. Facing Unpleasant Facts: Narrative essays. Boston, MA: Mariner Books; 2008. p. 29-37.

34. van Stralen D. Ambiguity. Journal of Contingencies and Crisis Management. 2015;23(2):47-53. doi: 10.1111/14685973.12082.

35. Weick KE. Enacted sensemaking in crisis situations Journal of management studies. 1988;25(4):305-17.

36. May RM. Biological populations with nonoverlapping generations: stable points, stable cycles, and chaos. Science. 1974;186(4164):645-7. Epub 1974/11/15. doi: 10.1126/science.186.4164.645. PubMed PMID: 4412202.

37. Dieterly DL. Problem Solving and Decisionmaking: An Integration. Moffett Field, California: NASA Ames Research Center, 1980 Contract No.: NASA Technical Memorandum 81191.

38. Orr GE. Combat Operations C3I: Fundamentals and Interactions. Maxwell Air Force Base, Montgomery, AL: Airpower Research Institute, USAF; 1983.

39. Pólya G. How to solve it: a new aspect of mathematical method. Garden City, N.Y.: Doubleday; 1945.

40. Neamy R. From Firescope to NIMS. Fire Rescue 2011;6(8).

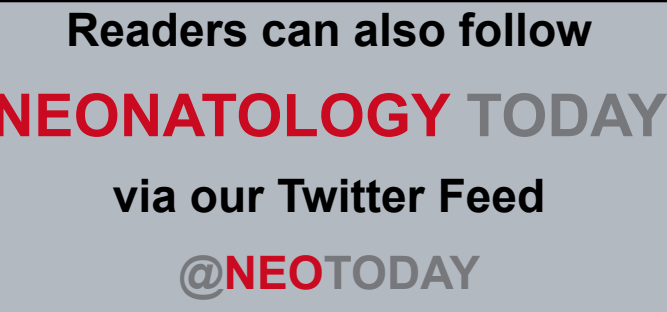

NEONATOLOGY TODAY

via our Twitter Feed
@NEOTODAY 
41. Cole D. The Incident Command System: A 25-Year Evaluation by California Practitioners. Emmitsurg, MD: National Fire Academy, 2000.

42. van Stralen D, Mercer TA. Pragmatic High-Reliability Organizations (HRO) Modulate the Functions of Stress and Fear Behaviors During Pandemic COVID-19: The Stress-FearThreat Cascade. Neonatology Today. 2020;15(10):126-34. doi: 10.51362/neonatology.today/2020101510126134.

43. Prud'homme A. Bob Bea, the Master of Disaster. Men's Journal 2013:72-5.

44. Boyd J. A discourse on winning and losing. Maxwell AFB, Alabama: Air University Press; 2018.

45. McConnell $M$, van Stralen D. Emergency medical decisionmaking in the tactical environment. The Tactical Edge (National Tactical Officers Association). 1997;15(3):32-9.

46. Weick KE, Sutcliffe KM, Obstfeld D. Organizing and the Process of Sensemaking. Organization Science. 2005;16(4):409-21. doi: 10.1287/orsc. 1050.0133.

47. Richards C. Boyd's OODA Loop (It's Not What You Think). In: Willeke E, editor. Proceedings of the Lean Software \& Systems Conference. Sequim, WA: Blue Hole Press; 2011. p. 127-36.

48. Boyd J. The Strategic Game of ? and ? In: Hammond GT, editor. A discourse on winning and losing. Maxwell AFB, Alabama: Air University Press; 2018. p. 255-314.

49. van Stralen $D$, Westmoreland $T$. Use of a visual five-point respiratory exam to evaluate breathing in the operational area. Special Operations Medical Association Scientific Assembly (SOMSA); December 8-11, 2014; Tampa, FL2014.

50. van Stralen D, Mercer TA. The Nature of Neonatal Experience during Pandemic COVID-19. Neonatology Today. 2021;16(3):87-97. doi: 10.51362/neonatology.today/202131638797.

51. Rasmussen J. What Can Be Learned from Human Error Reports? In: Duncan KD, Gruneberg MM, Wallis D, editors. Changes in Working Life. New York, NY: Wiley; 1980. p. 97-113.

Disclosures: The authors have no relevant disclosures

NT

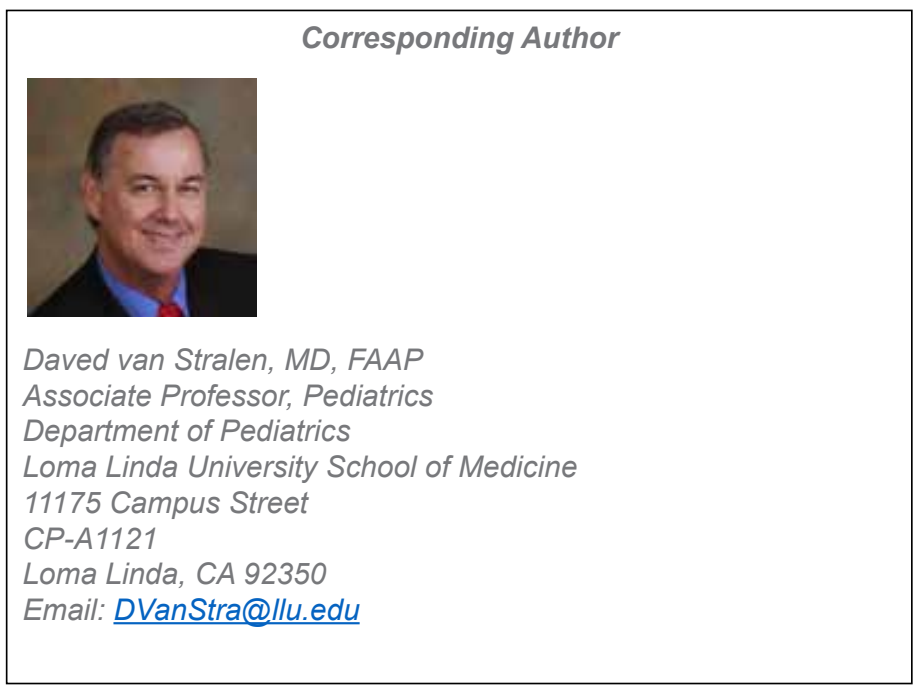

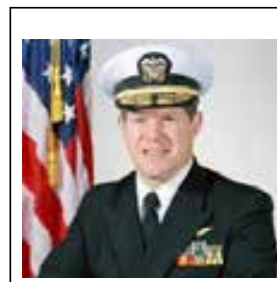

Thomas A. Mercer

Rear Admiral

United States Navy (Retired)

\section{Acknowledgments}

Karl Weick, Rensis Likert Distinguished University Professor of Organizational Behavior and Psychology, Emeritus, University of Michigan

Errol van Stralen, Ancora Education

William Gambino, CIV, DoD

Sean D. McKay, Element Rescue, LLC

Laura Elliott, Children's Subacute Center, Community Hospital of San Bernardin

William J. Corr, formerly with the Los Angeles City Fire Department, now deceased

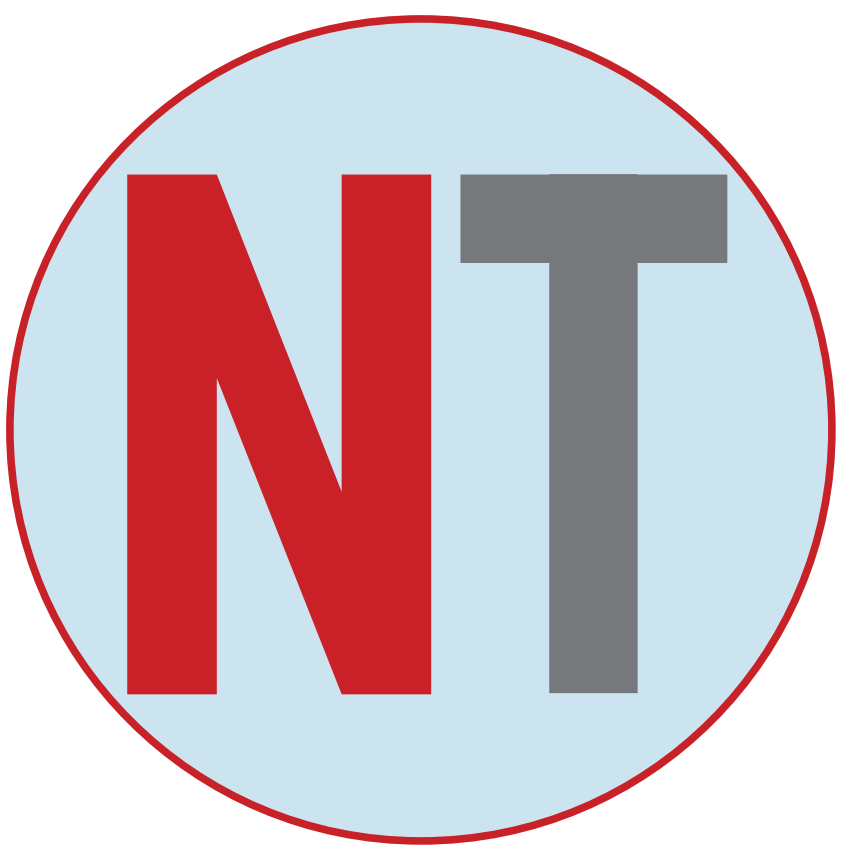

